MATEC Web of Conferences 40, 02018 (2016)

DOI: $10.1051 /$ matecconf/20164002018

C Owned by the authors, published by EDP Sciences, 2016

\title{
Research on the Combination of Underwater Acoustic Countermeasure Equipments Against Torpedo
}

\author{
MENG Jie ${ }^{1}$, WANG Yong Jie ${ }^{1}$, CAI Lei ${ }^{1}$, WEI Ran ${ }^{2}$ \\ ${ }^{1}$ Navy Submarine Academy, Shandong, Qingdao, 266041 \\ 2 PLA 91033 Troops, Shandong, Qingdao, 266035
}

\begin{abstract}
Today the use of acoustic countermeasure equipment has become the main means in submarine defense torpedo operation. Combination of acoustic countermeasure equipments are used during the operation so that we can amplify the countermeasure effect. Based on the subject of the acoustic countermeasure equipments' combined use, this paper analyses the interference between these soft kill countermeasure equipments including gas curtain, acoustic decoy and acoustic interferometer, summarizes the advantages and disadvantages of the different combined use of acoustic countermeasure equipments.
\end{abstract}

Keywords defense torpedo, underwater acoustic countermeasure, soft kill countermeasure equipment, combination use

\section{Introduction}

Underwater acoustic countermeasure is one of the key technologies of anti-torpedo. There are two kinds of underwater acoustic countermeasure equipment at present, soft kill countermeasure equipment and hard kill countermeasure equipment. The soft kill countermeasure equipment mainly includes acoustic self-guided decoy, acoustic interferometer, gas curtain and so on. The effect of anti-torpedo is very limited if using underwater acoustic countermeasure equipment separately. Combined use can observably increases the effect, but the effect of anti-torpedo also can be decreased by wrong combination of underwater acoustic countermeasure equipment. So correct combination of underwater acoustic countermeasure equipment is very important in anti-torpedo warfare. This article analyses mutual interference when multiple kinds of soft kill countermeasure equipment are used in combination, and summarizes the advantages and disadvantages of different combinations.

\section{Combined use of gas curtain and acoustic decoy}

In some situation, gas curtain will not only shield the submarine but also shield acoustic decoy when they are working together. When gas curtain is in the position between acoustic decoy and submarine, the torpedo's capability of detecting acoustic decoy will be decreased because of shield effect of gas curtain. Some methods that can deal with gas curtain shielding acoustic decoy are listed below when the way of gas curtain shielding submarine is not changed.

\subsection{Against passive acoustic guided torpedo}

The passive counter model of acoustic decoy is:

$\mathrm{SL}_{d}-T L\left(D_{d}\right)-I L \geq N L-D I+D T_{p}$

The third item IL in the formula is loss value of acoustic decoy's radiant intensity caused by gas curtain. Only when the formula holds, torpedo can detect acoustic decoy. So, incorrect usage of gas curtain can reduce the effect of acoustic decoy, what can cause worse antitorpedo effect.

\subsection{Against active acoustic guided torpedo}

In against active acoustic guided torpedo warfare, no matter the decoy is simulating constant intensity target, or fixed radiant intensity target, the torpedo will receive less signal power because of insert of gas curtain. Accordingly, the formula is:

$$
\begin{aligned}
& \mathrm{SL}-2 \mathrm{TL}\left(D_{d}\right)+T S_{d}-2 I L \geq N L-D I+D T_{a} \\
& \mathrm{SL}_{d}-T L\left(D_{d}\right)-I L \geq N L-D I+D T_{a}
\end{aligned}
$$

In addition, there is a question whether the decoy can detect radio pulses sent by torpedo. All kinds of decoy 
have threshold of detection. If the distance is far away from torpedo or the radiant intensity is too low, the energy of radio pulses sent by torpedo will too low to detect and analyze for decoy, what will course decoy can't response. Something should happen because of gas curtain: Decoy who should detect the torpedo's signal and correctly respond, now can't detect and respond because of gas curtain, so that it can't perform antitorpedo function. According to technic parameter of acoustic decoy and the passive sonar formula, it can be determine whether decoy can detect torpedo. When using gas curtain, parameter of acoustic decoy and torpedo need to be exchanged, that is:

$\mathrm{SL}-T L\left(D_{d}\right)-I L \geq N L_{d}-D I_{d}+D T_{d}$

In the formula, $\mathrm{SL}$ is torpedo sound level, $\mathrm{dB}$; $\mathrm{TL}(\mathrm{Dd})$ is transmission loss, $\mathrm{dB}$; IL is the loss coursed by gas curtain inserting, dB; NLd is decoy self-noise level, dB; Did is decoy receiving directivity indicator, $\mathrm{dB}$; DTd is acoustic decoy's threshold of detection, $\mathrm{dB}$.

If the formula holds, acoustic decoy can detect radio pulses sent by torpedo, formula (2), (3) is meaningful, otherwise decoy can't respond and is useless to interfere torpedo. Formula (2), (3) can't work.

When gas curtain is in the position between torpedo and decoy and the curtain size can hide the decoy, formulas above can work. Otherwise they can't be used. In addition, when gas curtain and decoy are used together, we thought torpedo's detection to gas curtain will not be affected by decoy.

\section{Combined use of gas curtain and acoustic interferometer}

In the situation of combined use of gas curtain and acoustic interferometer, if the position of gas curtain is between torpedo and acoustic interferometer, interfering effect will be decreased too. The processing on submarine echo and radiation noise level will remain the same if gas curtain shield submarine. While gas curtain shield acoustic interferometer, what will create torpedo interference noise level down.

$N L_{j}=\mathrm{SPL}-\mathrm{TL}\left(D_{j}\right)-\mathrm{IL}$

$\mathrm{SPL}-\mathrm{TL}\left(D_{j}\right)-\mathrm{IL} \geq \mathrm{NL}-\mathrm{DI}+\mathrm{DT}$

Because torpedo is keep moving, but gas curtain is unmovable, so interaction between gas curtain and acoustic interferometer will only happen in certain battle position. When relative position among torpedo, gas curtain and acoustic interferometer has changed because of torpedo's movement, gas curtain will be outside the sight of acoustic interferometer and torpedo; the interaction will not exist any longer.

\section{Combined use of gas curtain and acoustic decoy}

There are three possible parts of interaction, when combined use of gas curtain and acoustic decoy.

(1) Radiated noise of acoustic interferometer interferes in the torpedo detection of submarine.

(2) Acoustic interferometer interferes in torpedo detection of acoustic decoy.

(3) Acoustic decoy detection of radio pulses sent by torpedo will be interfered when acoustic interferometer is too close to acoustic decoy. More seriously, acoustic decoy can't distinguish torpedo detection radio pulses to respond.

The following formulas are used by torpedo active and passive detection of submarine.

$\mathrm{SL}-2 \mathrm{TL}+\mathrm{TS} \geq N L_{j}-D I(\theta)+D T_{a}$

$\mathrm{SL}-\mathrm{TL} \geq N L_{j}-D I(\theta)+D T_{p}$

Torpedo detection of decoy should put the effect of acoustic decoy into consideration. Right part of the formula above should be changed to $\operatorname{NLj-DI}(\theta)+\mathrm{DT}, \mathrm{DI}(\theta)$ is torpedo self-guarded receiving directivity function, $\mathrm{NLj}$ is torpedo interference noise level when acoustic interferometer is working.

$$
N L_{j}=\mathrm{SPL}-\mathrm{TL}\left(D_{j}\right)
$$

The influence on acoustic interferometer detection of acoustic decoy needs to consider formula(4), at this time:

$$
\mathrm{SL}-\mathrm{TL}\left(D_{d}\right) \geq N L_{d j}-D I(\theta)_{d}+D T_{d}
$$

In the formula, $\mathrm{DI}(\theta)$ is acoustic decoy receiving directivity function, NLdjis acoustic decoy interference noise level when acoustic interferometer is working.

$$
N L_{d j}=\mathrm{SPL}-\mathrm{TL}\left(D_{d j}\right)
$$

In the formula, SPL is radiation noise spectrum of acoustic interferometer, $\mathrm{dB}$; $\mathrm{TL}(\mathrm{Ddj})$ is transmission losses in the distance from acoustic interferometer to acoustic decoy, dB.

Also because of the acoustic interferometer is not all the same in different directions, so $\mathrm{DI}(\theta) \mathrm{d}$ is used to express acoustic decoy receiving directivity function. When torpedo and acoustic decoy are in the same location or the same line of bearing within acoustic decoy receive sector, $\theta=0, \mathrm{DI}(\theta) \mathrm{d}=0 \mathrm{~dB}$; otherwise, $\theta \neq 0, \mathrm{DI}(\theta) \mathrm{d} \neq 0 \mathrm{~dB}$; their values must be determined by acoustic decoy receiving directivity function. So, the model of acoustic interferometer interference active detection is:

$\mathrm{SL}-\mathrm{TL}\left(D_{d}\right) \geq N L_{d j}$

If inequality is established, the acoustic decoy can receive torpedo detection pulse, and work normally. Otherwise, the acoustic decoy can't respond correctly.

\section{Combined use of multiple acoustic decoys}


When multiple acoustic decoys are working together and the distance between each other is short, mutual interference between acoustic decoys would happen. What will cause response logic confuse and reduce the effect of anti-torpedo. Through analyzing of the work mechanism of acoustic decoy, we know that acoustic decoy only handles sound pulse and send them back, not detect where sound pulse comes from. So, if one decoy receives sound pulse sent by other decoy, it will respond the signal. At that time, decoy who is busy to respond can't detect sound pulse sent by torpedo. Effect of antitorpedo would become worse.

Signal energy function relationship between several decoys is need to be analyzed to determine whether two decoys will interfere with each other. According to passive sonar function:

$$
S L_{d 1}-\mathrm{TL}\left(D_{d d}\right) \geq N L_{d 2}-D I_{d 2}+D T_{d 2}
$$

In the formula, SLd1 is first decoy response emission sound level, dB; TL(Ddd) is one-way transmission loss, $\mathrm{dB}$; NLd2 is second decoy self-noise level, $\mathrm{dB}$; Did2 is second decoy receive direction index, $\mathrm{dB} ; \mathrm{DTd} 2$ is second decoy detection threshold value, $\mathrm{dB}$. If the distance between two decoys is close, the one-way transmission loss is small and formula (13) is established. Two decoys will respond each other and form closedloop interference.

\section{Summary}

Based on the above analysis, it must be consider that battle position, anti-torpedo equipment characteristics, target characteristics and so on to achieve good results. If using acoustic interferometer against passive guided torpedo, the acoustic interferometer will be detected by torpedo as a strong false target most probably. The torpedo will give priority to follow the acoustic interferometer. Then the torpedo may go through the acoustic interferometer to found submarine easily. The principle of using acoustic interferometer is try to put it between scanner and target to make a gas shell. When the torpedo found acoustic interferometer to false target, the decoy who can simulate radiated noise of submarine will be a strong fake target to against torpedo. The decoy has the ability of echo retransmission, which can respond torpedo active pulse detecting signal, add additional Doppler frequency shift and time delay. Self-propelled acoustic decoy can make the distance between torpedo and submarine longer, what can give submarine more time to escape. In order to achieve the ideal effect, acoustic decoy combinations and submarine movement ways are changed according to the different warning distance of incoming torpedo. All these will cause torpedo search target difficultly. So, combined use of different underwater acoustic countermeasure equipment in anti-torpedo Warfare is efficient.

\section{References}

1. Dj.M. Maric, P.F. Meier and S.K. Estreicher: Mater. Sci. Forum Vol. 83-87 (1992), p. 119

2. David Foxwell, Jane's Navy International,Torpedo defences on the alert[J], Forum Vol. 9 (2008) p. 113

3. Department of Defence, Defence Modeling and Simulation Office,High Level Architecture RunTime Infrastructure[S],1999

4. Armo,K.R.The Relationship between a submarine , s maximum speed and its evasive capability[D] Naval Postgraduate School, Monterey,CA, 2000

5. Ricardi, Christican Jeannin, "Albatros"torpedo alert system,UDT,2003 